DOI: $10.12731 / 2306-1561-2013-4-19$

\title{
FORGETTING MODEL OF EDUCATIONAL INFORMATION SYSTEM FOR TRAINING
}

\section{Stroganov V.Yu., Makarenko L.F., Yartcev M.I., Yagudaev G.G.}

\section{Abstract}

In the psychology of learning is defined as the absorption of trainees certain system of knowledge and skills. This requires a change in the external (physical) and internal (mental) activity (behavior). Education in psychology is defined as the process of stimulation and management of external activity the student as a result of which he has formed a certain knowledge, skills and abilities.

The processes of learning and teaching are discussed in various aspects: biological, physiological, social, philosophical, etc. The learning system requires a model of the object of study - the student model. Under the student model is meant representation of the process that takes place in the training as a result of his perception of the one or the other training information.

Keywords: learning, trained, forgetfulness, models, educational information, personnel.

\section{УДК 004.8:681.3}

\section{МОДЕЛИ ЗАБЫВАНИЯ УЧЕБНОЙ ИНФОРМАЦИИ ДЛЯ СИСТЕМЫ ПОДГОТОВКИ ПЕРСОНАЛА}

\section{Строганов В.Ю., Макаренко Л.Ф., Ярцев М.И., Ягудаев Г.Г.}

\section{Аннотация}

В психологии научение определяют, как усвоение обучаемым определенной системы знаний, умений, навыков. Это предполагает изменение внешней (физической) и внутренней (психической) деятельности (поведения). Обучение в психологии определяется как прочесс стимулящии и управления внешней активностью обучаемого, в результате которой у него формируются определенные знания, навыки и умения.

Прочессы научения и обучения рассматриваются в различных аспектах: биологическом, физиологическом, сочиальном, философском и др. В обучающей системе необходимо наличие модели объекта обучения - модели обучаемого. Под моделью обучаемого понимается представление того процесса, который проходит в обучаемом в результате восприятия им той, или иной обучающей информации.

Ключевые слова: научение, обучаемый, забывание, модели, учебная информация, персонал. 


\section{Процессы научения и обучения}

Исключительно важную роль в изучении процессов научения и обучения играет исследование памяти. Память является одним из важнейших психических процессов, реализующих усвоение знаний. Начало экспериментальной психологии памяти связано с опытами Г. Эббингауза. Он первый разработал количественные методы исследования запоминания и забывания. Материалом запоминания были бессмысленные слоги. Была построена кривая изменения объема памяти в зависимости от времени, прошедшего после запоминания, т.е. кривая времени забывания $[1,9]$. Эту кривую называют кривой забывания или сохранения. Она выражает объем памяти через разные промежутки времени в «процентах сбережения». При этом под объемом памяти (кратковременной) понимается наибольшее число единиц запоминаемого материала, которое может быть сразу воспроизведено при одном повторении. Что касается долговременной памяти, то измеряют число повторений, необходимых для запоминания и безошибочного воспроизведения предъявленного для запоминания материала, и объем памяти определяют как отношение числа запоминаемых символов к числу повторений. Кривая Г.Эббингауза - это объем памяти как функция времени:

$$
b=\frac{100 \cdot k}{(\log t)^{c}+k}
$$

где $\mathrm{b}$ - процент удержанного в памяти материала в момент эксперимента (или контроля) либо объем памяти в “процентах сбережения”; $\mathrm{t}$ - время с момента полного овладения материалом в часах; с и $\mathrm{k}$ - константы, получаемые методом наименьших квадратов по экспериментальным данным. Эксперименты [1, 9], связанные с научением путем «проб и ошибок», по выработке и исследованию затухания условных рефлексов у животных подтвердили закон Г. Эббингауза. Однако уровень сохранения при заучивании осмысленного материала всегда выше. Е.Н. Соколов рассмотрел общие основы кривых сохранения и показал, что «нет абсолютно “чистых кривых забывания" для смыслового и бессвязанного материала. Есть кривые забывания определенного количества качественно своеобразного материала» $[1,9]$.

После ранних работ Г. Эббингауза и Торндайка эмпирические данные, полученные при научении, стали выражать в виде уравнений, которые не опирались на какую-либо теорию, а лишь интерпретировали имеющиеся данные. Так, А. Шукаревым было выведено следующее уравнение:

$$
y=a-b \cdot e^{-c n}
$$

где у - усвоение, определяемое как число правильных воспроизведений (успехов) за единицу времени; $\mathrm{n}$ - число испытаний; a - предел усвоения при $\mathrm{n} \rightarrow \infty$; b и c - 
константы. Т.Робертсоном было предложено уравнение, полученное по аналогии с мономолекулярной автокаталитической реакцией:

$$
y=\frac{b \cdot e^{A \cdot n}}{c+e^{A \cdot n}},
$$

где у - усвоение; $\mathrm{n}$ - число испытаний; $\mathrm{A}=\mathrm{ab} ; \mathrm{a}$ и с - константы (параметры обучаемого); $\mathrm{b}$ - предел усвоения при $n \rightarrow \infty$. Л. Терстоун предложил следующую формулу (так называемый гиперболический закон обучения):

$$
y=\frac{a \cdot(n+c)}{(n+c)+b},
$$

где у - усвоение; $\mathrm{n}$ - число испытаний; а и с - константы; $\mathrm{b}$ - скорость научения.

Дальнейшее развитие применения математических методов к исследованию проблем научения связано с работами К. Халла [8]. Он ввел переменную, которую назвал «силой навыка». Она выражается формулой:

$$
H R S=M\left(1-e-b_{n}\right)
$$

где HRS - «сила навыка», или ассоциативная переменная, связывающая стимул и реакцию; M - асимптотическое значение «силы навыка»; b - параметр, выражающий скорость научения; $\mathrm{n}$ - число обучающих опытов (или проб с подкреплением). Модель Халла позволяет предсказывать результаты, получаемые при обучении парным ассоциациям.

В 40-50-е гг. в психологии обучение стали рассматривать как стохастический процесс. Психологи утверждали, что основной переменой в психологии должна быть вероятность ответа, или реакции. Р. Буш и Ф. Мостеллер, основываясь на том, что процесс научения является марковским, построили так называемые стохастические модели обучаемости. Примерно в то же время В. Эстес, К. Берг, Дж. Миллер, У. МакГилл и др. разрабатывали подобные стохастические модели, которые получили название «линейные модели обучения». При построении данных моделей вводится вероятность pn того, что обучаемый в $n-$ м испытании даст ответ $A_{l}$. Альтернативным является ответ $A_{2}$. Соответственно вероятность того, что обучаемый в n-м испытании даст ответ $A_{2}$, равна $1-p_{n}$. В каждом испытании обучаемый дает ответ, получая при этом какое-то подкрепление, например, узнает правильный ответ. В зависимости от подкрепляющего события $E_{j}$ в n-м испытании меняется вероятность ответа в n+1-м испытании: 


$$
p_{n}+1=a_{j p n}+b_{j}
$$

где параметры $a_{j}$ и $b_{j}$ увеличивают или уменьшают вероятность ответа. Эти параметры зависят от того, подкрепляет ли событие $E_{j}$ ответ $A_{l}$ или $A_{2}$. Так, в модели Буша-Мостеллера для случая двух альтернатив вводятся операторы:

$$
p_{n=1}=\left\{\begin{array}{lc}
\alpha_{1} p_{n}+\left(1-\alpha_{1}\right) \lambda_{1} & x \in A_{1} \\
\alpha_{2} p_{n}+\left(1-\alpha_{2}\right) \lambda_{2} & x \in A_{2}
\end{array}\right.
$$

где $-\lambda_{1}, \lambda_{2}\left(0 \leq \lambda_{1}, \lambda_{2} \leq 1\right)-$ неподвижные точки, т. е. если $p_{n}=\lambda_{i}(i=1,2)$, то $p_{n}+1=p_{n}$.

В. Эстесом была построена стохастическая модель для задачи обучения парным ассоциациям. Во время каждого опыта обучаемому представляется некоторый возбуждающий образ (стимул) и указывается его правильное наименование. Такое сочетание возбуждения и правильного ответа в психологии называют подкреплением. Во время проверочного испытания предъявляется только возбуждающий образ, на который обучаемый должен дать правильный ответ. Вводится следующая формализация. Пусть $E_{1}, E_{2}, \ldots E_{N}$ - элементы возбуждения; $A_{1}, A_{2}, \ldots A_{R}$ альтернативные ответы; $p_{i j, n}-$ вероятность того, что элемент возбуждения $E_{i}$ во время n-го опыта вызовет ответ $A_{j}$. Тогда процесс приобретения навыка описывается следующей функцией:

$$
p_{i j, n}=p_{i j, n}+c\left(1-p_{i j, n}\right) \text {, }
$$

где $c$ - константа $(0<c<1)$.

Еще в одной работе описанные выше модели Халла и Терстоуна интерпретируются в терминах стохастических моделей. Так модель Халла приобретает вид:

$$
p_{n}+1=p_{n}+(1-\alpha)\left(1-p_{n}\right)
$$

где $p_{n}$ - вероятность приобретения навыка (или правильного ответа) в $\mathrm{n}-\mathrm{M}$ испытании; $\alpha(0<\alpha<1)$ - константа. Сравнивая данную модель с моделью Эстеса, легко заметить, что она является частным случаем модели Эстеса.

В модели Терстоуна величина у интерпретируется как вероятность приобретения навыка $p_{n}=0$ при $n=1$ и стремящаяся к 1 при $n \rightarrow \infty$. Уравнение Терстоуна приобретает вид: 


$$
p_{n}=\frac{n-1}{n-1+b},
$$

где $b$ - скорость научения. В модели Рестла вероятность изменяется по следующему правилу:

$$
p_{n}=1-\frac{(1-\Theta)^{n-1}}{\Theta+(1-\Theta)^{n}},
$$

где $\Theta$ - константа.

В соответствии с гипотезой о том, что после начального периода обучения возникает «внезапная» обученность, лежащая в основе, так называемой теории «скачков». Вводится случайная величина хn, которая принимает значение 1 , если в nом испытании имеет место событие $A_{l}$, и $O$ - если в $n$-ом испытании имеет место событие $A_{2}$.

Тогда $p_{n}=P\left\{x_{n}=1\right\} \quad-\quad$ вероятность события $A_{l}, \quad q_{n}=P\left\{x_{n}=0\right\}=1-p_{n}$. Предположим, что обучаемый в начале опыта находится в некотором состоянии $S_{l}$, a, затем, после некоторого испытания nі переходит в состояние $S_{2}$ и остается в нем до конца эксперимента. В этом случае вероятность $p_{n}$ равно $p$, если обучаемый в $\mathrm{n}$-ом испытании находился в состоянии $S_{l}$, и 1 - если обучаемый в n-ом испытании находился в состоянии $S_{2}$.

Кроме того, вводится вероятность перехода из состояния $S_{1}$ в состояние $S_{2}$ на $n-$ м испытании:

$$
P\left\{S_{2} \mid S_{1} \text { npu } n-1\right\}=\beta
$$

где $\beta$ - некоторый параметр, $0 \leq \beta \leq 1$.

Дальнейшее развитие подобные модели получили в работах Р. Аткинсона, Г. Бауэра, Э. Кротерса. В настоящее время проблемы научения используются в статической теории обучения.

Пусть в момент времени $t=0$ обучающая информация воспринята учеником, а при $t>0$ ему задается вопрос по этому материалу. Если в момент $t=\tau$ обучаемый дает неправильный ответ на этот вопрос, то $\tau$ соответствует времени забывания. Предполагается, что время $\tau$ - непрерывная случайная величина с функцией распределения $P(t)=P\{\tau<t\}$. В случае экспоненциального распределения:

$$
P(t)=1-e-\lambda t
$$


где $\lambda$ - интенсивность забывания [1/c]. Среднее время забывания равно $1 / \lambda$ [c]. В эой работе для описания процесса забывания используются также распределения Вейбулла, Эрланга и Гамма-распределение.

\section{Процессы забывания}

Проблемам построения математических моделей некоторых характеристик памяти посвящен ряд работ. В одной из них исследуется длительность сохранения информации. При этом вероятность правильного ответа при отсроченном на время воспроизведении определяется по формуле:

$$
P \tau=P_{0} e-\gamma \tau
$$

где: $P_{0}$ - та же вероятность при немедленном воспроизведении; $\gamma$ - скорость забывания информации, определяемая методом наименьших квадратов.

Ряд работ посвящен исследованию процессов накопления и забывания информации и делается попытка их математического выражения. Пусть $\Delta \tau-$ длительность отдельного периода времени поступления информации, $i_{0}$ - средняя скорость восприятия информации обучаемым (в единицах информации в секунду). Так как в «кодирующее устройство мозга» вследствие потери части информации поступает некоторая ее доля $0<\sigma<1$, то количество информации, воспринятой обучаемым за время $\Delta \tau$, определяется как $\Delta J=\sigma_{0} \Delta \tau$. Суммарное количество накопленной информации определяется как:

$$
J=\sum^{n} \Delta J
$$

где $n$ - общее число периодов $\Delta \tau$. Однако, так как происходит потеря информации в результате ее забывания, то вводится некоторая поправка, учитывающая рассеивание приобретенной в момент $\tau$ порции информации до момента времени $\mathrm{t}$ как функция $K(t-\tau)$. Параметры данной функции и параметр $\sigma$ зависят от индивидуальных особенностей обучаемого. С учетом процесса забывания изменение количества информации в зависимости от времени t выражается в виде:

$$
J=\sum^{n} K(t-\tau) \cdot \sigma \cdot i_{0} \cdot \Delta \tau
$$

Исследования процесса научения и памяти были и остаются одними из главных проблем исследований в психологии и педагогике. Новая область обучения требует создания новых моделей [1 - 10]. 


\section{Список информационных источников}

[1] Исмоилов М.И. Подготовка и переподготовка персонала предприятий промышленного и транспортного комплексов с применением мобильных технологий: Монография / М.И. Исмоилов, А.Б. Николаев, А.В. Остроух.- SaintLouis, MO, USA: Publishing House Science and Innovation Center, 2013. - 166 c. ISBN 978-0-615-67111-6.

[2] Николаев А.Б., Толкаев Е.Ю. Эффективность использования технологий дистанционного обучения при повышении уровня знаний и навыков персонала промышленных предприятий. // Вестник Орловского государственного университета. Серия: Новые гуманитарные исследования. - 2010. - № 6. - С122123.

[3] Николаев А.Б., Толкаев Е.Ю. Дистанционное обучение как инновационный метод повышения квалификации персонала. // В мире научных открытий. - 2011. - № 9., - С. 80-85.

[4] Николаев А.Б., Строганов Д.В., Горячкин Б.С. Критерии оценки сложности учебной информации. // В мире научных открытий. - 2011.- № 9., - С. 7-15.

[5] Ягудаев Г.Г. Обоснование использования функций лагерра в задачах моделирования процессов научения-забывания // Автоматизация и управление в технических системах. - 2013. - № 1(3); URL: auts.esrae.ru/3-67 (дата обращения: 18.11.2013).

[6] Жажа Е.Ю. Рекуррентная схема генерации кусочно-функциональной зависимости процесса научения-забывания // ЭЛЕКТРОННОЕ ОБУЧЕНИЕ И ДИСТАНЦИОННЫЕ ОБРАЗОВАТЕЛЬНЫЕ ТЕХНОЛОГИИ. - 2013. - № 1; URL: eodot.esrae.ru/1-6 (дата обращения: 18.11.2013).

[7] Ягудаев Г.Г. Модели взаимной сцепленности процессов научения-забывания в системе переподготовки персонала // ЭЛЕКТРОННОЕ ОБУЧЕНИЕ И ДИСТАНЦИОННЫЕ ОБРАЗОВАТЕЛЬНЫЕ ТЕХНОЛОГИИ. - 2013. - № 1; URL: eodot.esrae.ru/1-7 (дата обращения: 18.11.2013).

[8] Суэтина Т.А., Ягудаев Г.Г., Жажа Е.Ю. Модели агрегирования оценочных показателей в системе аттестации персонала промышленных предприятий // Автоматизация и управление в технических системах. - 2012. - № 1; URL: auts.esrae.ru/1-47 (дата обращения: 18.11.2013).

[9] Остроух А.В. Информационные технологии в научной и производственной деятельности / [ред. А.В. Остроух] - М: ООО "Техполиграфцентр", 2011. - 240 с. ISBN 978-5-94385-056-1.

[10] Остроух, А.В. Принцип разработки учебных материалов для автоматизированных систем подготовки персонала нефтехимических предприятий / А.В. Остроух, А.М. Меркулов, П.А. Петриков, Ю.П. Бакатин // В мире научных открытий. Серия «Проблемы науки и образования». - 2012. - №2.6 (26). - С.184-193. 